\title{
CaM kinase II selectively signals to histone deacetylase 4 during cardiomyocyte hypertrophy
}

\author{
Johannes Backs, Kunhua Song, Svetlana Bezprozvannaya, Shurong Chang, and Eric N. Olson \\ Department of Molecular Biology, University of Texas Southwestern Medical Center, Dallas, Texas, USA.
}

\begin{abstract}
Class IIa histone deacetylases (HDACs) regulate a variety of cellular processes, including cardiac growth, bone development, and specification of skeletal muscle fiber type. Multiple serine/threonine kinases control the subcellular localization of these HDACs by phosphorylation of common serine residues, but whether certain class IIa HDACs respond selectively to specific kinases has not been determined. Here we show that calcium/ calmodulin-dependent kinase II (CaMKII) signals specifically to HDAC4 by binding to a unique docking site that is absent in other class IIa HDACs. Phosphorylation of HDAC4 by CaMKII promotes nuclear export and prevents nuclear import of HDAC4, with consequent derepression of HDAC target genes. In cardiomyocytes, CaMKII phosphorylation of HDAC4 results in hypertrophic growth, which can be blocked by a signal-resistant HDAC4 mutant. These findings reveal a central role for HDAC4 in CaMKII signaling pathways and have implications for the control of gene expression by calcium signaling in a variety of cell types.
\end{abstract}

\section{Introduction}

Changes in histone acetylation play a central role in the control of gene expression. Histone acetylation by histone acetyltransferases (HATs) relaxes the structure of nucleosomes and favors gene activation (1) whereas histone deacetylation by histone deacetylases (HDACs) promotes chromatin condensation, favoring transcriptional repression $(2,3)$. Association of HATs and HDACs with sequence-specific DNA-binding proteins allows for gene-specific activation and repression, respectively.

Over a dozen mammalian HDACs have been identified, which can be categorized into different classes based on sequence homology to 3 yeast HDACs and on structural characteristics (4). Class IIa HDACs, HDAC4, -5, -7, and -9, share a common structure, with a $\mathrm{C}$ terminal catalytic domain and an $\mathrm{N}$ terminal regulatory domain, that mediates interactions with transcription factors, coactivators, and corepressors (4). The $\mathrm{N}$ terminal regions of these HDACs contain a set of conserved serine residues that control their subcellular localization and confer signal responsiveness to downstream target genes (5-7). Phosphorylation of these sites creates binding sites for the 14-3-3 chaperone protein, which escorts phospho-HDACs from the nucleus to the cytoplasm, with consequent activation of HDAC target genes. In the nucleus, class IIa HDACs function as repressors of myocyte enhancer factor-2 (MEF2), a transcription factor that regulates muscle and stress-responsive genes (8-10). Interaction of MEF2 with the $\mathrm{N}$ terminal extension of class IIa HDACs silences the expression of MEF2 target genes (11).

Recent studies in knockout mice have identified class IIa HDACs as key regulators of tissue growth and development (reviewed in ref. 3). Mice lacking HDAC5 and HDAC9 show exag-

Nonstandard abbreviations used: AIP, autocamtide inhibitory peptide; ANP, atrial natriuretic peptide; Bis, bisindolylmaleimide I; CaMK, $\mathrm{Ca}^{2+} /$ calmodulin-dependent kinase; GST, glutathione-S-transferase; HDAC, histone deacetylase; MEF2, myocyte enhancer factor-2; MITR, MEF2-interacting transcription repressor; NLS, nuclear localization signal; NRVM, neonatal rat ventricular myocyte; PE, phenylephrine. Conflict of interest: The authors have declared that no conflict of interest exists. Citation for this article: J. Clin. Invest. 116:1853-1864 (2006). doi:10.1172/JCI27438 gerated hypertrophic growth of the myocardium in response to diverse stress stimuli $(12,13)$. Mice lacking HDAC4 die perinatally because of abnormal chondrocyte hypertrophy that results in ectopic and premature ossification of endochondral bones (14), and mice lacking HDAC7 die during embryogenesis from abnormalities in endothelial cell development (S. Chang and E.N. Olson, unpublished observations).

Phosphorylation of class IIa HDACs serves to connect extracellular stimuli with the genome by governing the expression of HDAC target genes. Protein kinase D (PKD) and various $\mathrm{Ca}^{2+} /$ calmodulindependent kinases (CaMKs) transmit signals from $G$ protein-coupled receptors to the regulatory phosphorylation sites in class IIa HDACs in a variety of cell types (15-22). Whether there is specificity among the different class IIa HDACs with respect to their responsiveness to upstream kinases remains to be determined.

In the present study, we investigated the potential role of CaMKII in the transmission of signals to each of the 4 class IIa HDACs. We show that CaMKII signals specifically to HDAC4 but not HDAC5 by associating with a unique kinase-docking site contained in HDAC4. Depending on its subcellular localization, CaMKII can either induce nuclear export or block nuclear import of HDAC4. Signaling by endogenous CaMKII is required for agonist-induced cytosolic accumulation of HDAC4 in cardiomyocytes. Our findings reveal a novel mechanism for transcriptional reprogramming in response to $\mathrm{Ca}^{2+}$ signaling and have implications for understanding the mechanism of action of CaMKII in a variety of cell types.

\section{Results}

CaMKII specifically induces cytosolic accumulation of HDAC4. To determine whether different class IIa HDACs might display specific responses to upstream kinases, we began by expressing the 4 class IIa HDACs - 4, 5, 7, and MEF2-interacting transcription repressor (MITR; a splice variant of HDAC9) - in COS cells with kinases implicated in HDAC phosphorylation, including PKD and different isoforms of CaMK. HDAC4, -5 and -7 were exported from the nucleus to the cytoplasm, and MITR, which lacks a nuclear export signal, was redistributed in the nucleus from a punctate to a homo- 


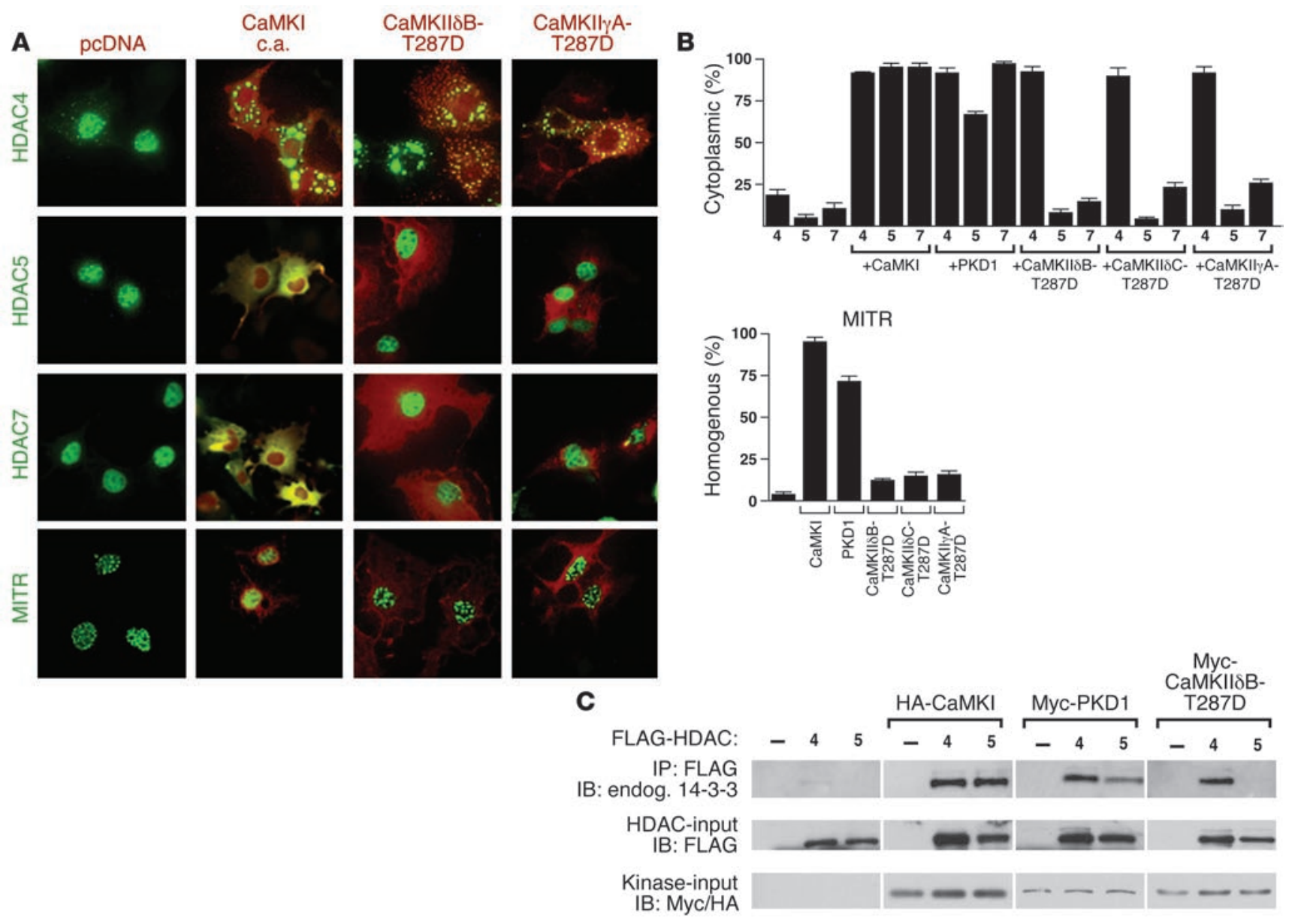

Figure 1

Selective response of HDAC4 to CaMKII. (A and B) COS cells were transfected with GFP-HDAC4, GFP-HDAC5, FLAG-HDAC7, or GFP-MITR

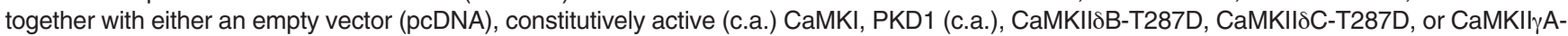
T287D. CaMKI c.a. induced nuclear export of all HDACs and changed the predominant nuclear localization of MITR from punctate to homogenous. CaMKIISB-T287D and CaMKIl $\gamma$ A-T287D selectively induced cytosolic accumulation of HDAC4 but did not affect the subcellular distribution of HDAC5, HDAC7, and MITR. (A) Representative images. Magnification, $\times 40$. (B) Quantitative analysis. (C) Coimmunoprecipitation assays with COS cell lysates were analyzed with an antibody directed against endogenous (endog.) 14-3-3 protein. HDAC-input, HDAC4 and -5 present in the COS cell lysate before IP was performed; Kinase-input, CaMKI, PKD1, or CaMKIISB-T287D present in the COS cell lysate before IP was performed.

geneous pattern in response to PKD, CaMKI, and CaMKIV (Figure 1, $\mathrm{A}$ and $\mathrm{B}$, and data not shown). Remarkably, however, constitutively active forms of CaMKII carrying point mutations (T287D) that mimic autoactivation caused the complete translocation of HDAC4 from the nucleus to punctate dots in the cytoplasm, but the other class IIa HDACs did not change their predominant nuclear localization in response to activated CaMKII $\delta \mathrm{B}$ and $-\delta \mathrm{C},-\gamma$, $-\beta$, or $-\alpha$ (Figure $1, A$ and $B$, and data not shown). However, HDAC7 appeared to display partial responsiveness to CaMKII.

To confirm that cytosolic accumulation of HDAC4 reflected its phosphorylation, we assayed for the association of HDAC4 with 14-3-3, which correlates with phosphorylation of class IIa HDACs $(3,6,15)$. FLAG-HDAC4 immunoprecipitates were analyzed by Western blot analysis using an antibody against endogenous 14-3-3 protein. Consistent with our immunocytochemical results, we observed that CaMKIIסB induced 14-3-3 binding to HDAC4 but not HDAC5 whereas PKD1 and CaMKI acted on both class IIa HDACs, suggesting that the latter enzymes act as general class IIa HDAC kinases (Figure 1C).
HDAC4 colocalizes with activated CaMKII. The CaMKIIS splicing variant $\mathrm{B}$ contains a nuclear localization signal (NLS) and thus likely targets nuclear proteins (23). Unexpectedly, activated CaMKIISB, which was generated by a T287D mutation (CaMKIISB-T287D), localized predominantly to the cytosol, in contrast to the WT (inactive) form, which was mainly localized to the nucleus (Figure 2A). Phosphorylation ofS332 next to the NLS ( ${ }_{328} \mathrm{KKRKSS}_{333}$ ) ofCaMKIISB has been shown to induce nucleocytoplasmic shuttling of CaMKIISB $(23,24)$. Consistent with these findings, the additional S332A

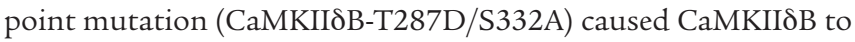
remain in the nucleus (Figure $2 \mathrm{~A}$ ).

The observation that CaMKIISB-T287D is predominantly cytosolic while HDAC5 is exclusively nuclear in unstimulated cells raised the possibility that the insensitivity of HDAC5 to CaMKII might reflect its sequestration in a different subcellular compartment than CaMKII. We therefore coexpressed the constitutively active and nuclear CaMKIISB-T287D/S332A mutant with HDAC4 and HDAC5. Like CaMKII-T287D, this mutant enzyme also induced cytosolic accumulation of HDAC4 but not 
A

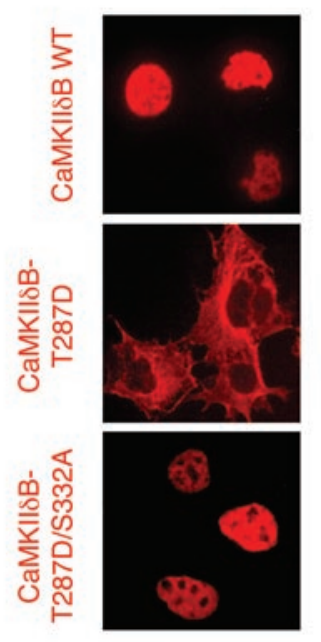

C

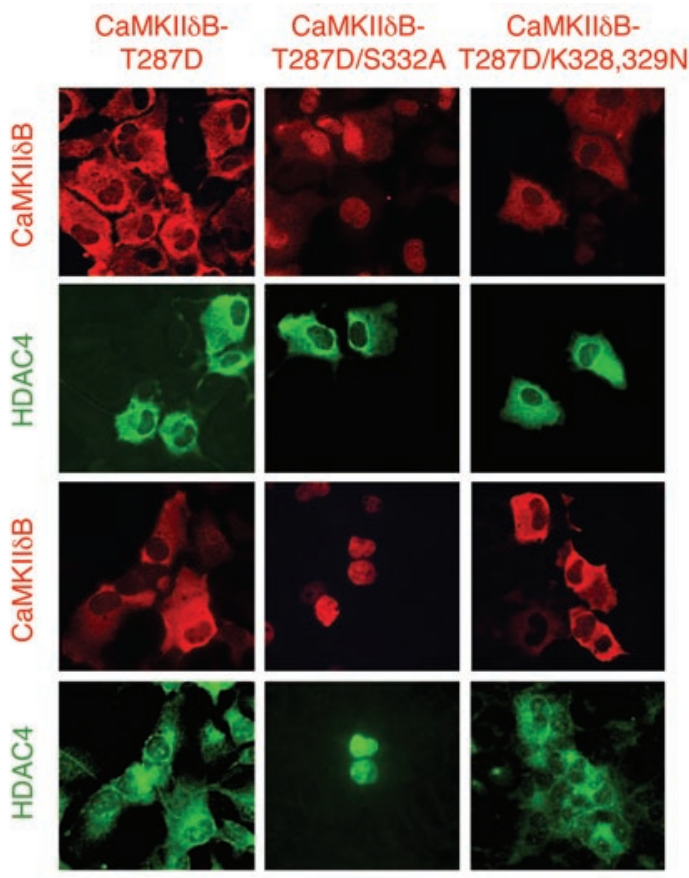

D

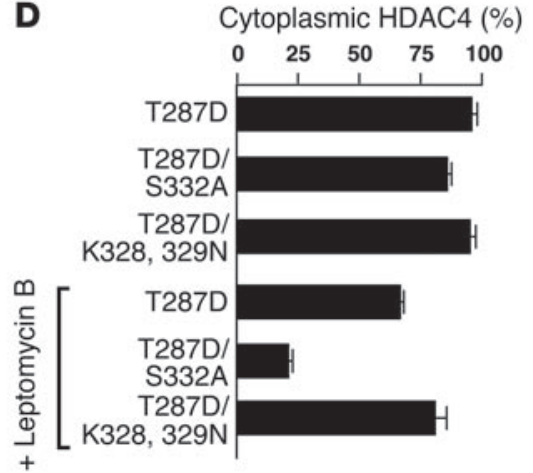

B
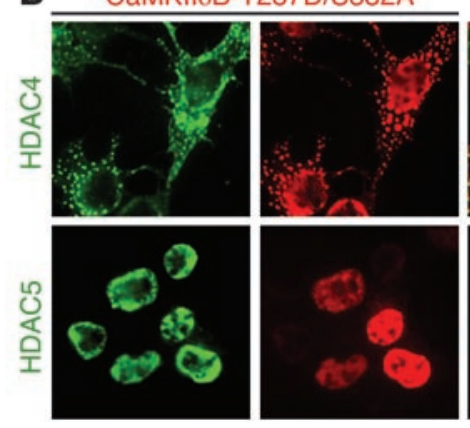

Merge

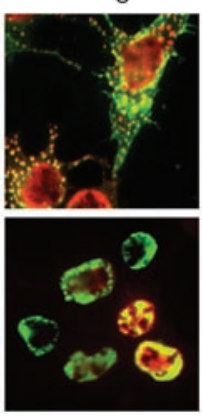

\section{Figure 2}

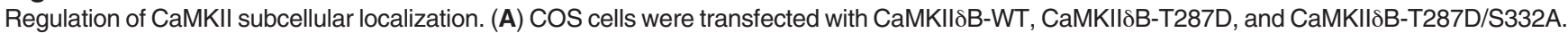
In contrast to the WT kinase, CaMKIISB-T287D localized predominantly to the cytosol. Substitution of S332 rendered CaMKII8B-T287D constitutively active and nuclear. (B) COS cells were cotransfected with CaMKII8B-T287D/S332A and HDAC4 or HDAC5. Note that only HDAC4 was exported in response to the double CaMKIIIB mutant and colocalized with the kinase. (C and D) COS cells were first transfected with the indicated CaMKIIIB mutants and 12 hours later with HDAC4. Twelve hours after transfection with HDAC4, cells were treated for another 4 hours either with $1 \mathrm{nM}$ leptomycin B (lower panel) or the vehicle ethanol (upper panel). Note that with leptomycin B, HDAC4 only accumulates in the nucleus in the presence of CaMKIIIB-T287D/S332A, which is active and nuclear, but not in the presence of CaMKIIIB-T287D or CaMKIIIB-T287D/K328,329N, which are active and cytosolic. (A-C) Representative images. Magnification, x40. (D) Quantitative analysis of experiment shown in C.

HDAC5 (Figure 2B). Although CaMKIISB-T287D/S332A cannot be phosphorylated next to its NLS and is clearly localized to the nucleus when expressed in the absence of HDAC4, it colocalized with HDAC4 to the cytosol (see Figure 2B), suggesting a possible physical interaction between HDAC4 and CaMKII that results in coshuttling of the 2 proteins to the cytosol.

CaMKII induces nuclear export and blocks nuclear import of HDAC4. The above findings raised the question of how the predominantly cytosolic CaMKIISB-T287 mutant causes accumulation of HDAC4 to the cytosol. The same phenomenon was observed for the CaMKII splice variants A and C, CaMKII $\alpha A$ and CaMKII $\beta$ 'e, which do not contain an NLS but which exerted the same effect on HDAC4 localization as CaMKIISB (data not shown). In addition, a CaMKIISB mutant in which the NLS was destroyed (T287D/K328,329N) (23) and that localized exclusively to the cytosol, caused HDAC4 to colocalize and accumulate to the cytosol (Figure 2C).

Because HDAC4 was located in the cytosol in approximately $20 \%$ of cells under basal conditions (Figure 1B), we hypothesized that it might cycle between the nucleus and the cytosol and that cytosolic forms of CaMKII might block nuclear import of HDAC4. Therefore, we examined the effect of leptomycin $B$, an inhibitor of CRM1-dependent nuclear export, on the intracellular redistribution of HDAC4 in response to different active CaMKIISB mutants (T287D, which is predominantly cytosolic; T287D/S332A, which is exclusively nuclear; and 
A

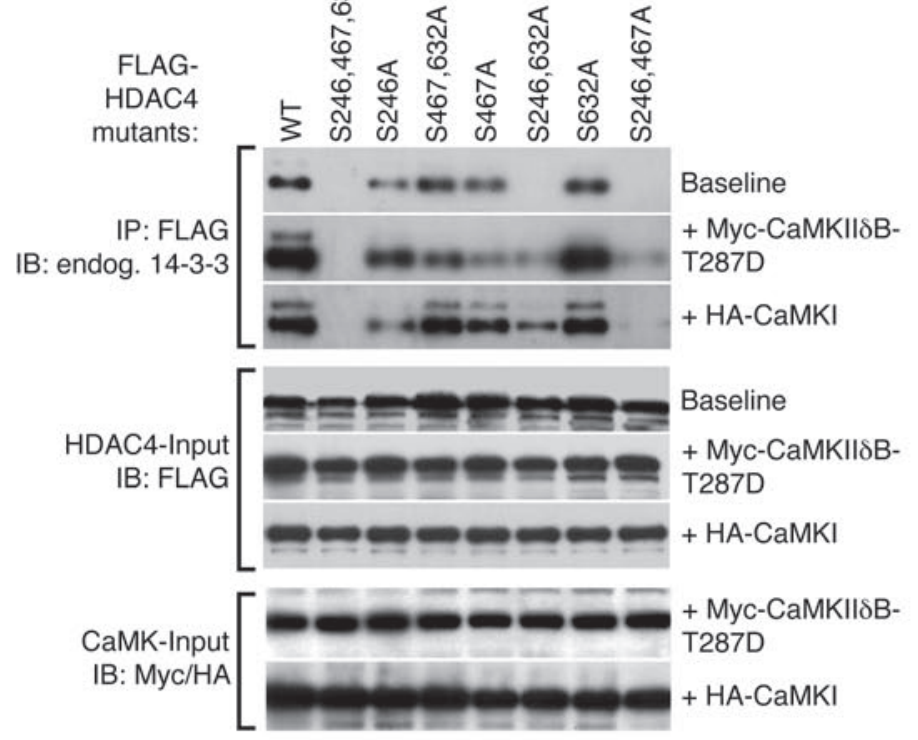

B

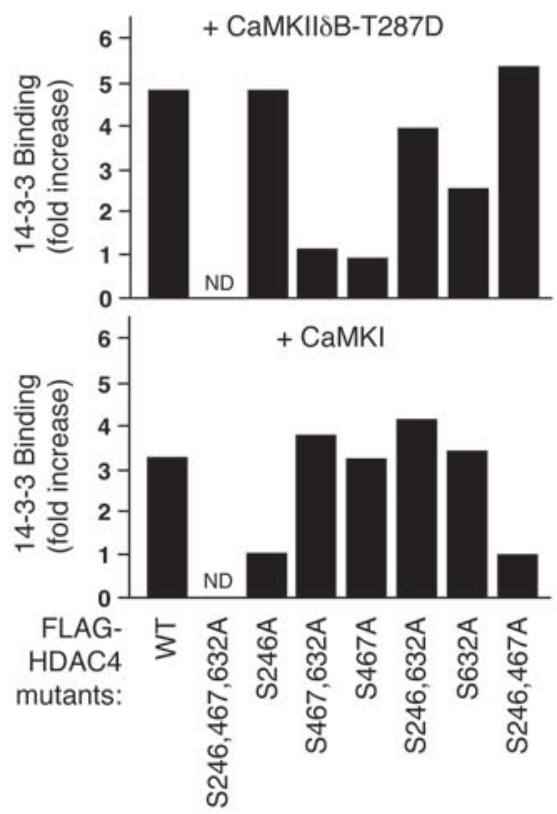

C

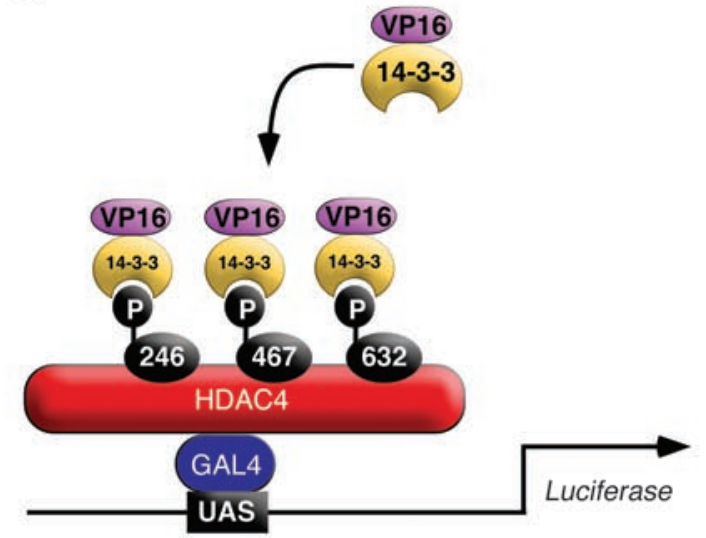

D

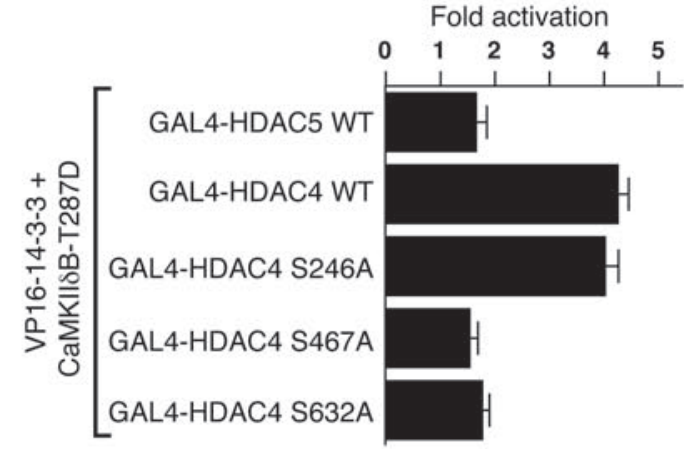

E

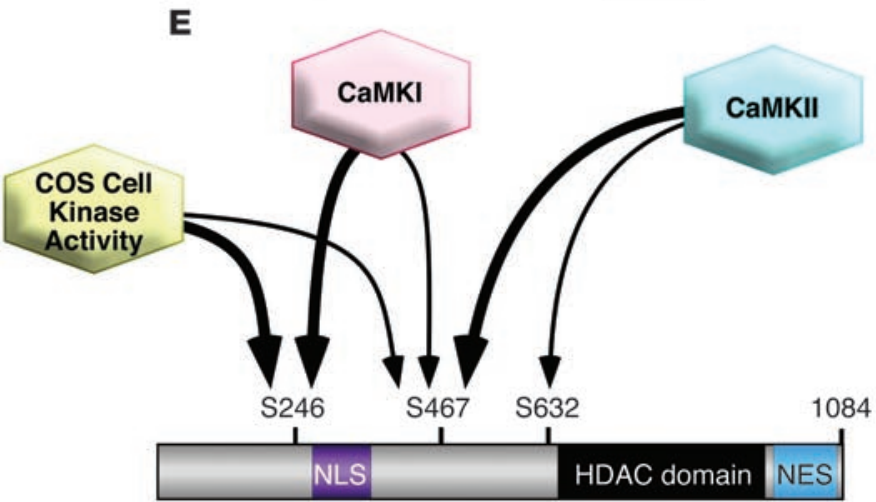

Figure 3

Detection of 14-3-3 binding sites of HDAC4 in response to CaMKI and CaMKII. (A and B) Coimmunoprecipitation assays with COS cell lysates were analyzed with an antibody directed against endogenous 14-3-3 protein. The effects of CaMKI and CaMKII on various FLAG-HDAC4 mutants were tested. HDAC4-input, HDAC4 present in the COS cell lysate before IP was performed; CaMK-input, CaMKI or CaMKIIIB-T287D present in the COS cell lysate before IP was performed. (B) Fold-increase in 14-3-3 binding in response to CaMKII or CaMKI as compared with baseline. (C) The N terminal half of HDAC4 (amino acids 1-740) was fused to the GAL4 DNA-binding domain, and 14-3-3 was fused to the VP16 transcription activation domain. If GAL4-HDAC4 is not phosphorylated, it cannot recruit VP16-14-3-3 and cannot activate the GAL4-dependent luciferase reporter. (D) As indicated, different GAL4-HDAC constructs were used in this assay in the absence and presence of CaMKIIIB-T287D. COS cells were transfected with the indicated constructs. Increase in 14-3-3 binding is expressed as compared with control conditions without kinase. (E) CaMKI and CaMKII phosphorylation sites of HDAC4 are shown. ND, not detectable; NES, nuclear export signal.

T278D/K328,329N, which is exclusively cytosolic) (Figure 2, $\mathrm{C}$ and $\mathrm{D})$. All these mutants are constitutively active and all induced cytosolic accumulation of HDAC4 in the absence of leptomycin B. After short-term treatment with leptomycin B (4 hours, $1 \mathrm{nM})$, HDAC4 accumulated in the nucleus only after stimulation with nuclear CaMKIISB-T287D/S332A, suggesting that this mutant induced nuclear export of HDAC4. In contrast, in the presence of cytosolic CaMKIISB-T287D and -T287D/ $\mathrm{K} 328,329 \mathrm{~N}$, leptomycin B failed to induce nuclear accumulation of HDAC4, indicating that these mutants block nuclear import. Thus, even when CaMKII is in the cytosol, it can phosphorylate HDAC4, favoring derepression of HDAC target genes. 
Analysis of HDAC4 phosphorylation sites by 14-3-3 binding in response to CaMKI and CaMKII. To assess whether CaMKII uses different phosphorylation sites on HDAC4 than CaMKI, we performed additional coimmunoprecipitation experiments with endogenous 14-3-3 protein in COS cells. Consistent with previous work by others (5), the replacement of S246, S467, and S632 with alanine (mutant S246,467,632A) abolished 14-3-3 binding at baseline as well as in the presence of constitutively active CaMKI or CaMKII (Figure 3A). Whenever S246 was mutated (alone or in combination with S467 or S632), baseline 14-3-3 binding and CaMKI-induced 14-3-3 binding were impaired (Figure 3, A and B). Conversely, the S467,632A mutant was still phosphorylated at baseline and sensitive to CaMKI, identifying S246 as the major phosphorylation site for the endogenous COS cell kinase activity and CaMKI. In contrast, whenever S467 was mutated, CaMKII-induced 14-3-3 binding was impaired, but the S246,632A mutant was still sensitive to CaMKII, identifying S467 as the major phosphorylation site for this kinase (Figure 3, A and B). CaMKI could also phosphorylate S467 to a modest degree, but its phosphorylation of S632 was almost undetectable. In contrast, CaMKII efficiently phosphorylated S632. Although 14-3-3 binding to the S467,632A mutant was readily detectable, there was no increase above baseline in response to CaMKII, indicating that S246 is not a CaMKII site.

These data were confirmed using a mammalian 2-hybrid assay that detects the specific association of 14-3-3 fused to VP16 (transcriptional activation domain) with the $\mathrm{N}$ terminal half of HDAC4 (amino acids 1-740) fused to GAL4 (DNA-binding domain) (Figure 3C). A similar system with HDAC5 was successfully used to detect HDAC kinases in a high-throughput expression screen (25). Phosphorylation of HDAC4 by CaMKII\&B-T287D created docking sites for VP16-14-3-3, resulting in activation of the GAL4-dependent reporter (Figure 3C). In contrast, CaMKIISB-T287D failed to activate the reporter in the presence of GAL4-HDAC5 and VP16-14-3-3, confirming the selective responsiveness of HDAC4 to CaMKII and establishing this system as a reliable method for detecting 14-3-3 binding to HDAC4. In this assay, we replaced each of the 3 signal-responsive serines (S246, S467, and S632) with alanines. Disruption of S246 did not affect the interaction of 14-3-3 with HDAC4 in response to CaMKIIסB-T287D (Figure 3D). Consistent with our 14-3-3 coimmunoprecipitation data, disruption of S467 or S632 reduced 14-3-3 binding. Taken together, the results of the direct and indirect 14-3-3-binding assays demonstrate that CaMKI preferentially phosphorylates S246 and S467, whereas CaMKII phosphorylates S467 and S632 on HDAC4 to induce 14-3-3 binding, as schematized in Figure 3E.

Mapping a CaMKII-responsive region of HDAC4. To further examine the molecular basis for the selective responsiveness of HDAC4 to CaMKII, we generated mutant constructs encoding chimeric HDAC4/HDAC5 proteins. As shown in Figure 4A, only those chimeric proteins containing residues 529-657 of HDAC4 were responsive to CaMKIIסBT287D. Because this region contains S632, we asked whether this phosphorylation site determines the selective responsiveness of HDAC4 to CaMKIISB-T287D. The amino acid sequence surrounding S632 of HDAC4 differs at 4 positions from the corresponding region in HDAC5, which surrounds S661. We mutated 3 of these residues in addition to 2 differing amino acids in the consensus sequence around S498 of HDAC5 to those of HDAC4 (HDAC5 S494G/S499A/G657S/ T659A/A665S). Despite these changes, this HDAC5 mutant was still nonresponsive to CaMKII (Figure 4B). These results suggest that differences in the CaMKII phosphorylation sites of HDAC5 were insufficient to account for its insensitivity to CaMKII.
Activated CaMKII directly interacts with a unique domain of HDAC4. Based on the observation that CaMKII-T287D colocalized with HDAC4, we performed immunoprecipitation experiments to determine if the proteins interacted. As shown in Figure 4C, we found that CaMKII-T287D bound strongly to HDAC4 but showed almost no detectable interaction with HDAC5. Whereas weak binding of CaMKII to HDAC5 was detectable under low stringency conditions in the immunoprecipitation buffer, it was not detectable under high stringency conditions. However, CaMKII still bound strongly to HDAC4 under high stringency conditions, suggesting that HDAC4 possesses a unique domain that mediates a strong interaction with the kinase. Interestingly, we observed that activated CaMKII-T287D but not inactive WT CaMKII strongly bound to HDAC4 (Figure 4D). This finding suggests that autophosphorylation induces a conformational change in CaMKII, which allows it to bind to HDAC4.

Coimmunoprecipitation experiments using deletion mutants of HDAC4 delineated the CaMKII-binding domain to amino acids 585-608 of HDAC4 (Figure 4, E and F). Although HDAC4 shares extensive amino acid homology with other class IIa HDACs throughout its length, this CaMKII-binding region is not homologous to other class IIa HDACs (Figure 5A).

To pinpoint the residue(s) required for interaction of HDAC4 with CaMKII, we systematically mutated the residues in the minimal CaMKII-binding domain to alanines and tested the mutants for their ability to bind CaMKII by coimmunoprecipitation. As shown in Figure 5, A and B, substitution of R601 by alanine or phenylalanine markedly disrupted the physical interaction between HDAC4 and CaMKII.

To test whether R601 of HDAC4 was required for CaMKII responsiveness, we examined the subcellular localization of 2 HDAC4 mutants (R601A and R601F) in the presence of CaMKIISB-T287D. In contrast to WT HDAC4, these mutants only partially accumulated in the cytosol in response to CaMKIISB-T287D (Figure 5, C and D). Moreover, these HDAC4 mutants did not colocalize with CaMKIIסBT287D. In contrast, HDAC4-R601A and R601F mutants were still responsive to CaMKI (Figure 5D), indicating that R601 is specifically required for CaMKII sensitivity.

GAL4-HDAC4 mutants containing alanine, phenylalanine, lysine or leucine in place of R601 were markedly impaired in their ability to bind VP16-14-3-3 in response to CaMKIISB-T287D (Figure 5E) whereas the basal 14-3-3-binding activity of these HDAC4 mutants was comparable to that of WT HDAC4 (not shown). While HDAC4-R601A and R601K showed a slight CaMKIIinduced increase in 14-3-3 binding (below 2-fold), mutations of R601 to nonpolar hydrophobic amino acids (phenylalanine or leucine) prevented 14-3-3 binding in response to CaMKII completely. Again, these CaMKII nonresponsive mutants were not affected in their ability to bind 14-3-3 in response to CaMKI (not shown).

To further test whether phosphorylation of HDAC4 by CaMKII is dependent on a direct interaction between these 2 proteins, we purified a bacterially expressed HDAC4 protein fragment (amino acids 419-670), which contains the CaMKII phosphorylation sites S467 and $\mathrm{S} 632$ and the CaMKII docking site as a glutathione-S-transferase (GST) fusion protein (GST-HDAC4-WT). When incubated together

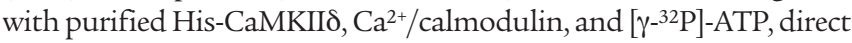
phosphorylation was observed (Figure 5F). In contrast, phosphorylation of GST-HDAC4-R601F was 7-fold weaker, indicating that full responsiveness of HDAC4 to CaMKII depends on stable docking of the kinase on HDAC4. In this assay, active WT CaMKII enzyme was used, and HDAC4 phosphorylation was abolished by $\mathrm{Ca}^{2+}$ depletion 
A

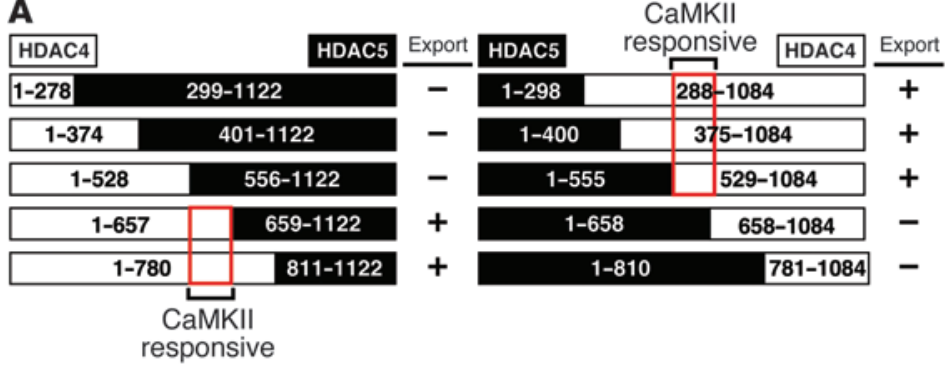

B

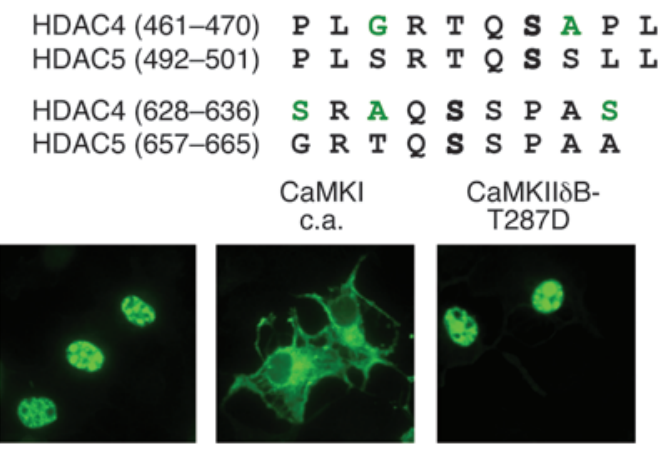

HDAC5 S494G/S499A/G657S/T659A/A665S

C

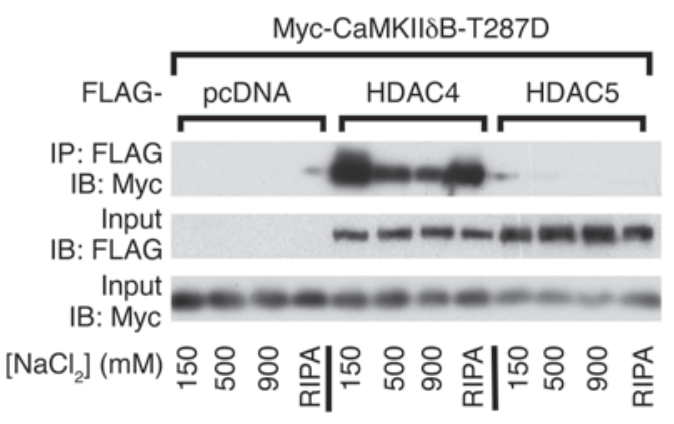

D

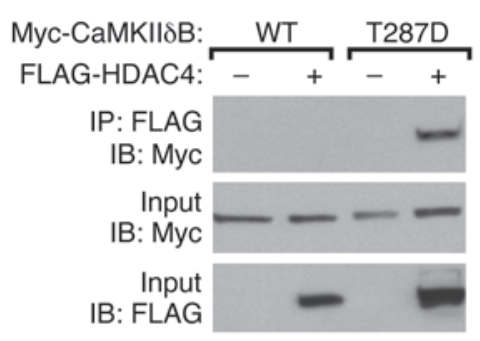

Myc-CaMKII\&B-T287D

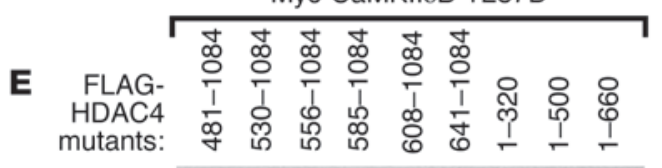

IP: FLAG

IB: Myc

Input

IB: Myc

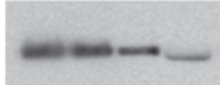

Input

IB: FLAG

F MEF2binding domain

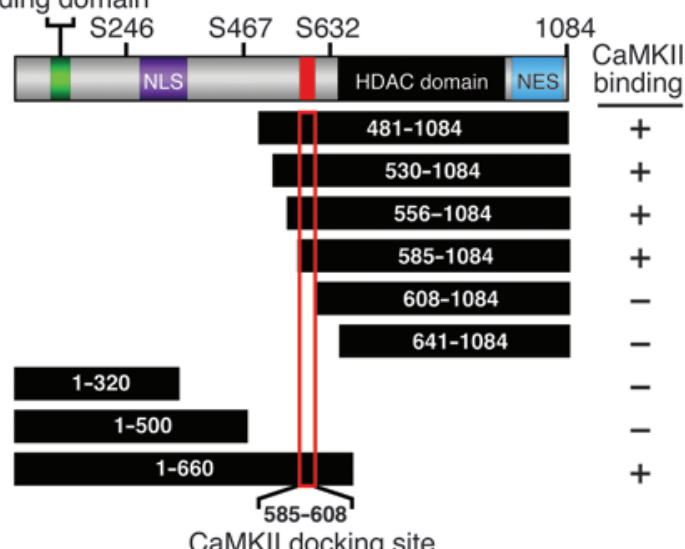

Figure 4

Mapping the CaMKII-responsive region of HDAC4. (A) Chimeric HDAC4/5 proteins (as indicated) were expressed in COS cells in the presence of CaMKIISB-T287D. Subcellular localization was verified by immunocytochemistry. Amino acids 529-657 were revealed to be required for cytosolic accumulation of HDAC4 in response to CaMKIISB-T287D. (B) An HDAC5 mutant, in which the CaMKII consensus sites were mutated to the corresponding sites in HDAC4, was expressed in COS cells alone or with CaMKI c.a. and CaMKIIIB-T287D. This mutant was responsive to CaMKI but not to CaMKIIIB-T287D. Magnification, $\times 40$. (C-F) Coimmunoprecipitation assays with COS cell lysates. (C) COS cells were cotransfected with FLAG-pcDNA, -HDAC4, or -HDAC5 and Myc-CaMKII8B-T287D. Various stringency conditions of the immunoprecipitation buffer were tested as indicated. Only HDAC4 binds strongly to CaMKII. (D) Cotransfection of FLAG-HDAC4 with either WT or constitutively activated Myc-CaMKII $\delta B-$ T287D. Only the activated form of CaMKIISB physically interacted with HDAC4. (E) IP of FLAG-HDAC4 deletion mutants coexpressed with Myc-

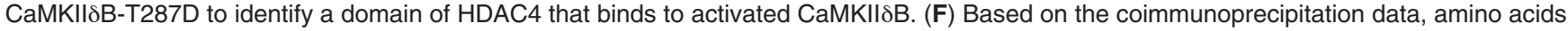
585-608 of HDAC4 were identified to be required for physical interaction with CaMKIISB-T287D and, therefore, define a CaMKII docking site.

with EGTA, confirming that phosphorylation of HDAC4 by CaMKII is not artificially caused by the T287D point mutation but that it also occurs with endogenously activated CaMKII.

CaMKII binds directly to HDAC4 because the same GST-HDAC4 fusion protein used in the in vitro kinase assay pulled down about $30 \%$ of active His-CaMKIII that was coincubated in the same tube (Figure 5G). The GST-HDAC4-R601F mutant did not pull down His-CaMKII $\delta$. The same result was obtained with in vitro-translated $\left[{ }^{35} \mathrm{~S}\right]-\mathrm{CaMKII \delta B}-\mathrm{T} 287 \mathrm{D}$ (not shown).
These findings raise the question of why CaMKIISB-T287D still induces some cytosolic accumulation of HDAC4-R601F (to about $50 \%$ ) (Figure 5D). We imagine 3 explanations for this phenomenon: (a) CaMKII activates an endogenous kinase in COS cells that induces cytosolic accumulation of HDAC4 independent of CaMKII docking; (b) there may be residual binding of CaMKII to the HDAC4R601F mutant, which is indetectable in the assay but sufficient to induce weak phosphorylation of HDAC4; (c) stable binding may not be absolutely essential for HDAC4 phosphorylation by CaMKII 
A

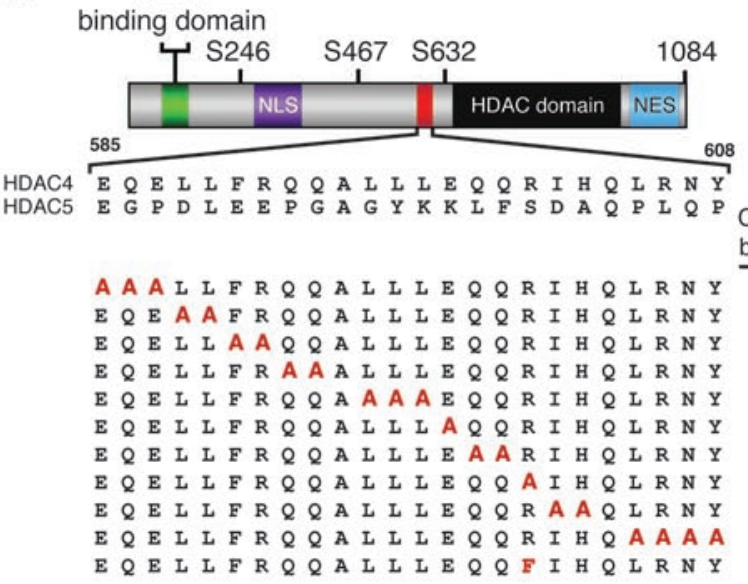

B

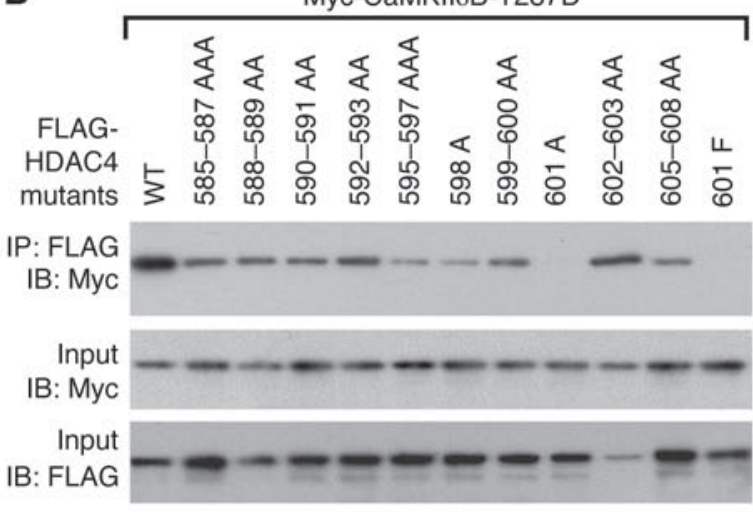

D

\begin{tabular}{l} 
CaMKII \\
binding \\
\hline+ \\
+ \\
+ \\
+ \\
+ \\
+ \\
+ \\
+ \\
+ \\
+
\end{tabular}

E

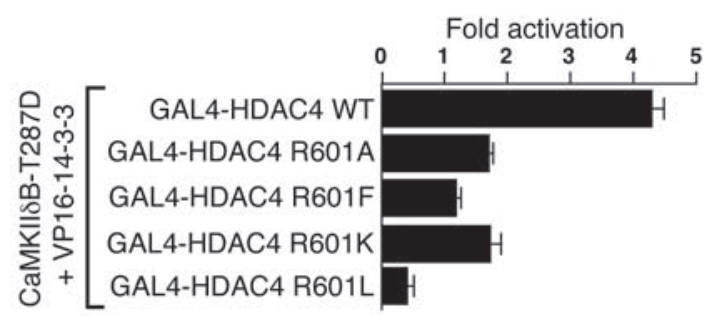

$\mathbf{F}$
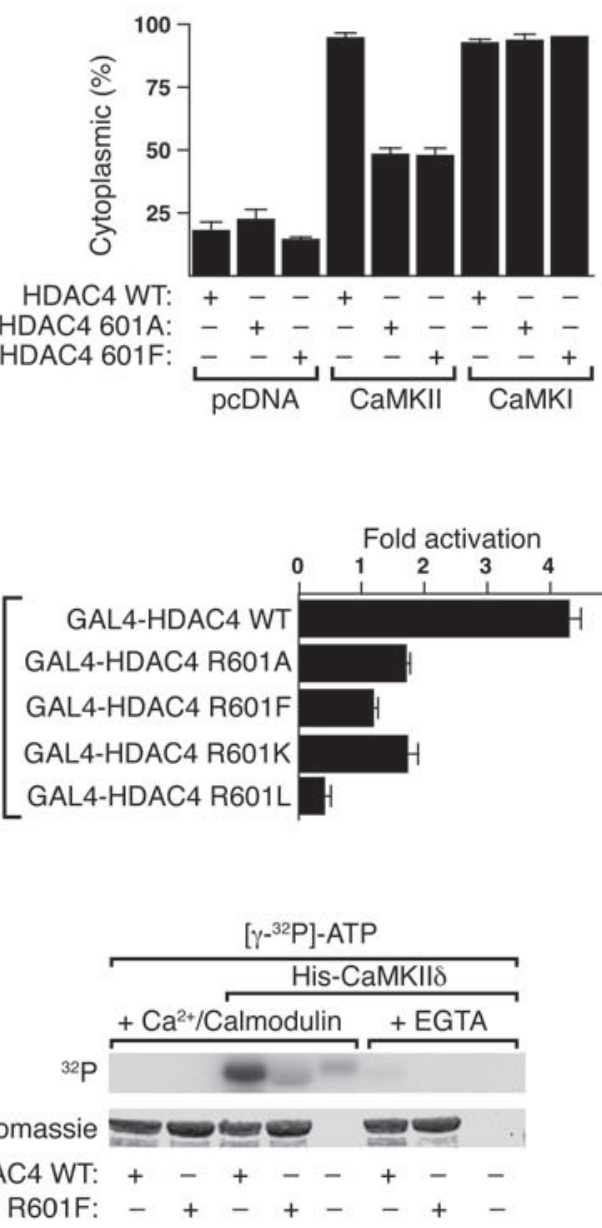

C

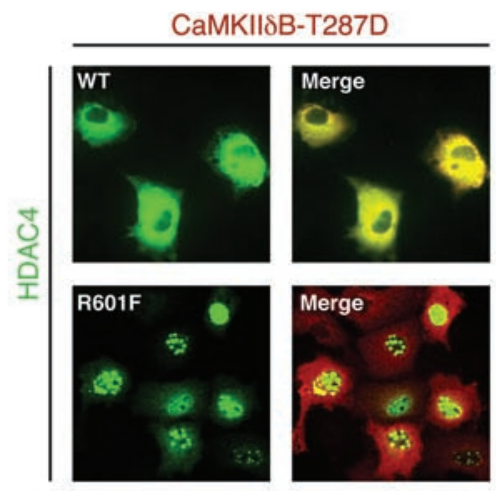

G

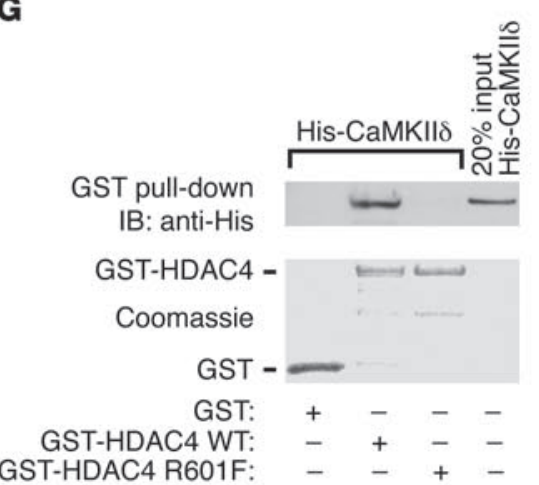

H

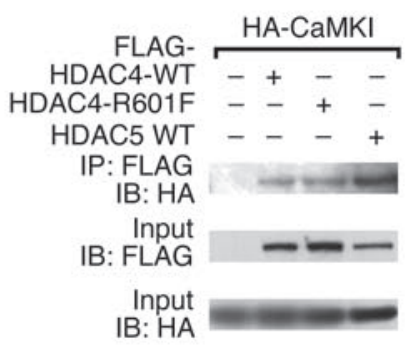

Figure 5

R601 of HDAC4 is required for full responsiveness to CaMKIISB-T287D. (A and B) FLAG-HDAC4 mutants carrying point mutations in the CaMKII docking region were coexpressed with Myc-CaMKIISB-T287D in COS cells. Coimmunoprecipitation revealed that substitution of R601 with

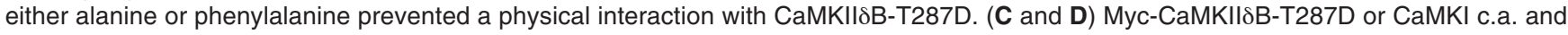
either FLAG-HDAC4-WT, -R601A, or -R601F were coexpressed in COS cells, and HDAC4 localization was determined 1 day after transfection. HDAC4-R601A and -R601F were still responsive to CaMKI but not to CaMKII8B-T287D and did not colocalize with the kinase. (C) Representative images. Magnification, ×40. (D) Quantitative analysis. (E) Mammalian 2-hybrid assay with GAL4-HDAC4 mutants and VP16-14-3-3. Substitution of R601 with phenylalanine and leucine prevented and with alanine and lysine markedly attenuated 14-3-3 binding. (F) In vitro kinase assay. Active His-CaMKII induced phosphorylation of GST-HDAC4-WT (amino acids 419-670) but not of the GST-HDAC4-R601F (419-670) mutant. Ca ${ }^{2+}$-depletion by EGTA prevented HDAC4 phosphorylation by CaMKII. (G) GST pull-down assay with GST, GST-HDAC4WT (419-670), GST-HDAC4-R601F (419-670) mutant, and active His-CaMKIIס. (H) Coimmunoprecipitation revealed that HA-CaMKI docks to FLAG-HDAC4-WT and -R601F to the same degree, however, it docks to HDAC5 with greater affinity. 
A

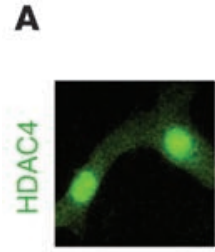

B
$+\mathrm{PE}$

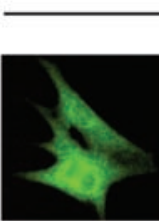

HDAC4 localization
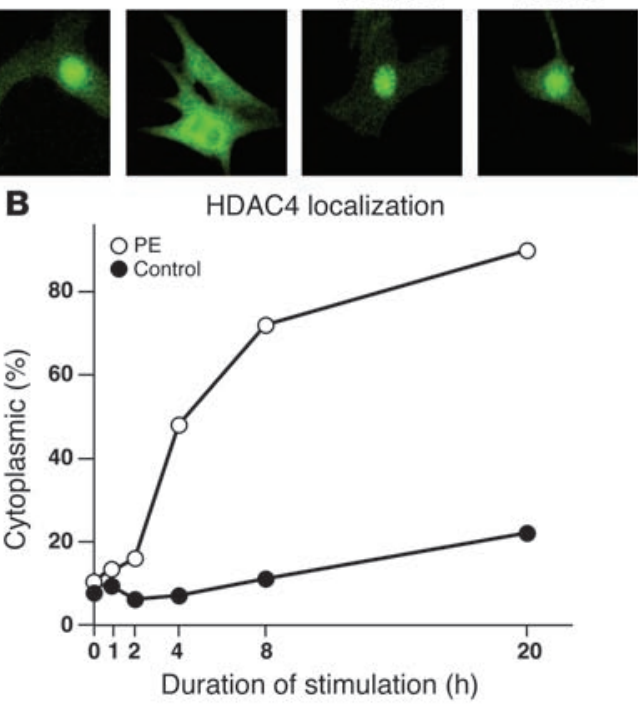

+ Bis

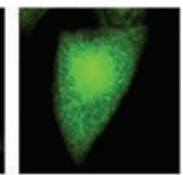

C

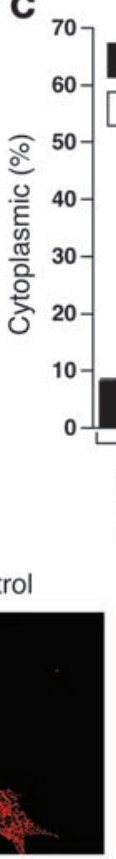

E

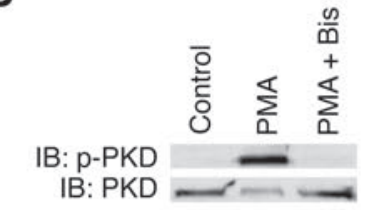

$\mathbf{F}$

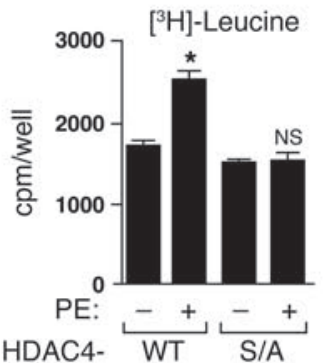

Figure 6

Cytosolic accumulation of HDAC4 in cardiomyocytes. (A-C) NRVMs were infected with adenoviral FLAG-HDAC4 or GFP-HDAC5. Subcellular distribution of HDAC4 and HDAC5 was verified following stimulation with PE $(20 \mu \mathrm{M})$ for 4 hours. NRVMs were pretreated with the kinase inhibitors staurosporine (Stauro; $500 \mathrm{nM})$, KN93 $(5 \mu \mathrm{M})$, KN62 $(10 \mu \mathrm{M})$, AIPII-2 (500 nM), Bis $(2.5 \mu \mathrm{M})$, Gö6976 (200 nM), or H89 (1 $\mu \mathrm{M})$. (A) Representative images. (B) Quantitative analysis of time-dependent PE-induced cytosolic accumulation of HDAC4. (C) Effects of kinase inhibitors on PE-induced cytosolic accumulation of HDAC4. (D) NRVMs were treated with PMA with and without Bis. Immunoblotting was performed with antibodies against PKD (lower panel) and phospho-S744/S748 PKD (p-PKD; upper panel). (E-G) NRVMs were infected with adenoviruses encoding FLAG-HDAC4-WT or FLAG-HDAC4-S246,467,632A (FLAG-HDAC4-S/A). One day after infection, cells were grown in serum-free media for 24 hours and then stimulated with PE $(20 \mu \mathrm{M})$. Cells were fixed and stained with anti-sarcomeric $\alpha$-actinin (red signal; 24 hours after PE) (E) or anti-ANP (perinuclear green signal; 12 hours after PE) (G). HDAC4-infected NRVMs were identified by anti-FLAG staining (green in $\mathbf{E}$ or red in $\mathbf{G}) .(\mathbf{F})\left[{ }^{3} \mathrm{H}\right]$-leucine was added to NRVMs 2 hours after PE stimulation and $\left[{ }^{3} \mathrm{H}\right]$-leucine incorporation was measured 24 hours later. ${ }^{*} P<0.05$ vs. WT without $P E$ and vs. S/A with PE. NS, not significant vs. S/A without PE. (A, E, and G) Representative images were captured at a magnification of $\times 40$. 


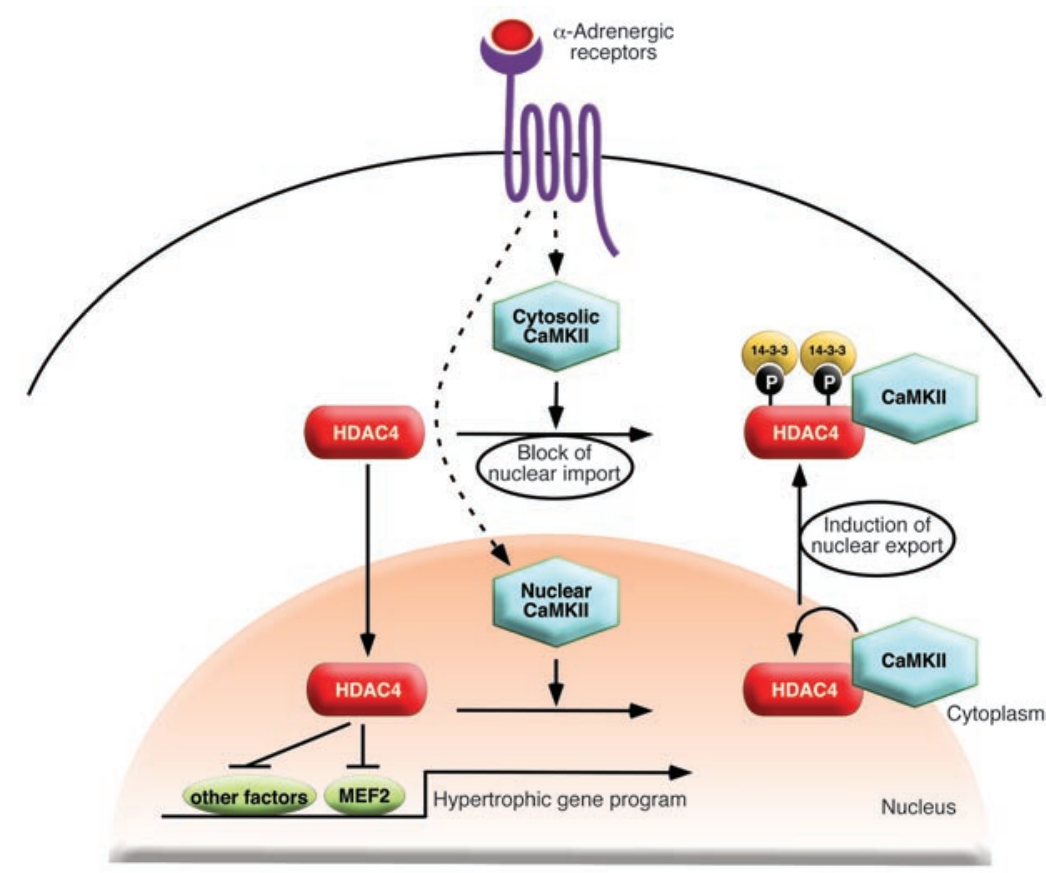

Figure 7

A model of CaMKII-dependent cytosolic accumulation of HDAC4. $\alpha$-Adrenergic stimulation activates CaMKII. Activated CaMKII interacts with HDAC4, which results in phosphorylation of HDAC4 and 14-3-3 protein-mediated nuclear export or a block of nuclear import. Cytosolic accumulation of HDAC4 derepresses MEF2 or other transcription factors, which associate with the nonphosphorylated form of HDAC4 when CaMKII is inactive.

if CaMKII can act inefficiently through a "kiss-and-run" type of mechanism. Evidence for the latter 2 possibilities is provided by the in vitro kinase assay (Figure $5 \mathrm{~F}$ ) in which CaMKII induced slight phosphorylation of HDAC4-R601F (7-fold less than to the WT fragment) although no direct binding was detected (Figure 5G).

Another question one could ask is whether other kinases, which act as general class IIa HDAC kinases, such as CaMKI, also require docking to their substrates. Indeed, coimmunoprecipitation of HDAC4 and HDAC5 with CaMKI confirmed that these 2 proteins interact (Figure 5H). The HDAC4-R601F mutation did not affect CaMKI binding to HDAC4, suggesting that CaMKI uses a different docking site than CaMKII. Further studies are needed to characterize the binding domains of other class IIa HDAC kinases, such as CaMKI, CaMKIV, and PKD.

$\alpha$-Adrenergic agonists signal to HDAC4 via CaMKII. A variety of neurohumoral signals have been shown to induce HDAC5 to translocate from the nucleus to the cytoplasm of cardiomyocytes as a consequence of PKD-dependent phosphorylation of 14-3-3-binding sites in its $\mathrm{N}$ terminal regulatory domain (16). To determine whether HDAC4 was regulated in a similar manner, we examined its subcellular distribution in neonatal rat ventricular myocytes (NRVMs) exposed to phenylephrine (PE), a potent hypertrophic agonist that acts through the $\alpha$-adrenergic receptor. As shown in Figure 6, A and B, PE caused a time-dependent cytosolic accumulation of HDAC4.

Cytosolic accumulation of HDAC4 in response to PE was markedly reduced by the general serine/threonine kinase inhibitor staurosporine as well as by the CaMKII inhibitors KN93, KN62, and autocamtide inhibitory peptide II-2 (AIPII-2). In contrast,

\section{Discussion}

bisindolylmaleimide I (Bis), Gö6976, and H89, which inhibit PKC, PKD, and protein kinase A, respectively, did not affect HDAC4 localization (Figure 6, A and C). Consistent with previous findings that the PKC-PKD axis regulates $\operatorname{HDAC5}(16,18)$, PE-induced nucleocytoplasmic shuttling of HDAC5 was not affected by KN93 (Figure 6A). The effectiveness of Bis was confirmed by the observation that it was able to prevent phosphorylation of the PKC phosphorylation sites on endogenous PKD (S744 and S748) in response to stimulation with PMA (Figure 6D), confirming that, despite the marked inhibition of PKD, PE-induced cytosolic accumulation of HDAC4 was caused by a PKC/PKD-independent signaling pathway, which we conclude from these results is the CaMKII pathway.

Adenoviral overexpression of WT HDAC4 did not prevent features of PE-induced cardiomyocyte hypertrophy, as assessed by the sarcomeric organization of NRVMs (Figure 6E), $\left[{ }^{3} \mathrm{H}\right]$-leucine incorporation (Figure $6 \mathrm{~F}$ ), and atrial natriuretic peptide (ANP) immunostaining (Figure 6G), because it is signal responsive and accumulated in the cytosol. In contrast, an HDAC4 mutant, in which the signal-responsive serines were replaced with alanines (S246,467,632A), stayed in the nucleus and blocked the hypertrophic response to $\mathrm{PE}$, suggesting that cytosolic accumulation of HDAC4 is essential for cardiomyocyte hypertrophy in response to CaMKII signaling (Figure 6, E-G).

Extracellular signals can communicate with the genome via regulated phosphorylation of class IIa HDACs, which results in their sequestration in the cytoplasm and activation of HDAC target genes. The results of this study identify HDAC4 as a specific and direct target for CaMKII signaling. HDAC4 contains a unique CaMKII docking site not found in other HDACs, which allows it to bind and respond specifically to CaMKII. These findings provide new insight into the mechanisms whereby $\mathrm{Ca}^{2+}$-dependent signals regulate cardiac growth and have implications for CaMKII signaling in other cell types.

Signal-dependent regulation of class IIa HDACs. Class IIa HDACs share a common structure, with a $\mathrm{C}$ terminal catalytic domain and an $\mathrm{N}$ terminal regulatory domain, that mediates interactions with transcription factors, coactivators, and corepressors and serves as a target for phosphorylation by CaMK and PKD (4). Upon phosphorylation, the 14-3-3 chaperone protein binds class IIa HDACs and mediates cytosolic localization $(5,15)$. Our findings demonstrate that stimulation of $\alpha$-adrenergic receptors induces cytosolic accumulation of HDAC4 via CaMKII. CaMKII isoforms are cytosolic and nuclear, raising the question of whether CaMKII induces nuclear export or blocks nuclear import $(5,6)$. By using CaMKII mutants, which localize exclusively to the cytosol or the nucleus, and by using an inhibitor of the nuclear export machinery, leptomycin B, we showed that activated CaMKII phosphorylates HDAC4 in both of these cell compartments, resulting in its sequestration in the cytoplasm (Figure 7).

How can $\mathrm{Ca}^{2+}$ signals activate nuclear CaMKII? While $\mathrm{Ca}^{2+}$ and calmodulin can translocate into the nucleus (26), there is also growing evidence that $\mathrm{Ca}^{2+}$ signals can be generated within the 
nucleus. For example, inositol 1,4,5-triphosphate $\left(\mathrm{InsP}_{3}\right)$ receptors have been identified on the nuclear membrane in ventricular myocytes $(27,28)$, suggesting that the nuclear envelope might serve as a pool for release of $\mathrm{Ca}^{2+}$, which could bind calmodulin associated with the nuclear membrane and in turn activate nuclear variants of CaMKII. Three alternative splicing variants of the CaMKII subunits that contain NLSs have been identified ( $\alpha \mathrm{B}, \delta \mathrm{B}$, and $\gamma \mathrm{A})(23)$. The critical role of alternative splicing of CaMKII in cardiac function has recently been demonstrated in mice lacking the essential splicing factor ASF/SF2 (29), which express the sarcolemmal

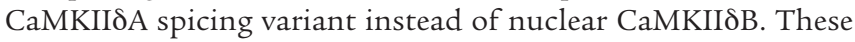
mice develop a hypercontraction phenotype with biventricular dilation. Interestingly, CaMKIISB is among the splicing variants that are upregulated in cardiac hypertrophy (30).

Our finding that activated CaMKII stably associates with HDAC4 even after cytosolic accumulation suggests that the stoichiometry between CaMKII and HDAC4 might have an important influence on the degree of cytosolic accumulation of HDAC4. Therefore, in addition to $\mathrm{Ca}^{2+} /$ calmodulin-dependent activation, upregulation of CaMKII expression, as described for CaMKIIJ and $\gamma$ in experimental heart failure (30), would be expected to markedly affect cytosolic accumulation of HDAC4.

Specificity of CaMK signaling. Signaling by CaMK has been implicated in a broad range of cellular, developmental, and physiological processes, including learning and memory, neurotransmission, maturation of oocytes, myocyte contractility, and muscle growth and gene expression, each of which requires phosphorylation of specific substrates within different subcellular compartments (31). Much remains to be learned about the molecular basis for the specificity of cellular responses to CaMK (and other kinases), particularly with respect to the mechanisms for signaling by the kinase to the genome that result in short- and long-term changes in gene expression. The complexity of CaMK signaling is underscored by the existence of 6 different CaMK genes (CaMKI, II $\alpha$, $I I \beta, I I \gamma, I I \delta$, and $I V)$, which give rise to multiple protein variants through alternative splicing (32).

Specificity of signaling events can be achieved by scaffold proteins, which mediate compartmentalization of kinases and phosphatases, as described, in general, for A-kinase anchoring proteins (33) and, in the case of CaMKII, for $\alpha$-kinase anchoring proteins (34). A distinct but related mechanism involves the tethering of the kinase to its substrate at a site that is distinct from the phosphorylation site. In this study, we identified a unique CaMKII docking site on HDAC4 that is not present on other HDACs. The docking site (amino acids 585-608) is located in relatively close proximity to the sites of HDAC4, which we show to be phosphorylated by CaMKII (S467 and S632). Interestingly, only the active form of CaMKII, mimicked by the T287D point mutation, interacts with HDAC4, suggesting that autophosphorylation induces a conformational change in CaMKII that enables it to bind HDAC4. Consistent with this notion, it has been demonstrated that highaffinity docking of CaMKII to the NMDA-type glutamate receptor requires autophosphorylation of CaMKII (35).

CaMKII and cardiac bypertrophy. There is increasing evidence for the involvement of CaMKII in pathological cardiac hypertrophy $(30,36-44)$. Inhibition of CaMKII in mice by overexpression of a CaMKII inhibitory peptide in the heart blocks adverse myocardial remodeling in response to $\beta$-adrenergic stimulation and myocardial infarction, providing strong evidence that CaMKII is required for a pathological hypertrophic response (38). CaMKII has also been implicated in endothelin-1-induced cardiomyocyte hypertrophy and in ANP gene expression during hypertrophy (39-41). Moreover, forced overexpression of calmodulin or CaMKII in the heart is sufficient to induce cardiac hypertrophy (40, 42-44). However, the downstream mechanisms and targets of CaMKII signaling that lead to changes in cardiac gene regulation have remained elusive.

CaMKII exists as a multimer consisting of $6-12 \alpha, \beta, \delta$, or $\gamma$ subunits, each encoded by a different gene $(31,32)$. Whereas CaMKII $\alpha$ and $\beta$ are mainly expressed in neuronal tissues, CaMKII $\delta$ and $\gamma$ are abundant in the heart and upregulated in clinical and experimental heart failure $(30,36,37)$.

Convergence of multiple protein kinases on class IIa HDACs. We and others have shown that PKD acts as a direct kinase of HDAC5 and -7 and induces their translocation from the nucleus to the cytoplasm (16-18). Here, we show that PKD acts as a general class IIa HDAC kinase like CaMKI and CaMKIV because it also changes the subcellular localization of HDAC4 and MITR. It is interesting to note that the catalytic domain of PKD is most homologous to that of CaMK (45). Why are there so many kinases for regulating class IIa HDACs? Given the expression of class IIa HDACs in a variety of cell types and the importance of $\mathrm{Ca}^{2+}$-signaling in modulating cellular phenotypes, it is intriguing to speculate that class IIa HDACs have evolved to use the same regulatory phosphorylation sites to respond to different physiologic stimuli and regulate distinct sets of genes in different cell types. In this regard, HDAC7 was shown to undergo cytosolic accumulation in Tlymphocytes in response to PMA-mediated activation of $\operatorname{PKD}(17,18$, 46). Similarly, HDAC4 and -5 are exported from the nucleus in hippocampal neurons in response to spontaneous activity, and HDAC5 is similarly controlled in cerebellar granule neurons subjected to depolarizing potassium (47). Moreover, electrical stimulation of skeletal muscle slow fibers has been shown to induce nucleocytoplasmic shuttling of HDAC4 but not HDAC5 (21). Thus, it seems that different cell types utilize different signaling pathways $\left(\mathrm{Ca}^{2+}\right.$ dependent through CaMK or $\mathrm{Ca}^{2+}$ independent through $\mathrm{PKD}$ ) to activate distinct gene programs.

Three studies seem to contradict the results of the present report because they show that CaMKII regulates the subcellular localization of HDAC5 in cerebellar granule and hippocampal neurons as well as in adult rabbit ventricular myocytes $(28,47,48)$. However, these studies do not address the molecular mechanism whereby CaMKII regulates HDAC5 nuclear export nor do they demonstrate direct phosphorylation of HDAC5 by CaMKII. The discrepancies between those studies and ours might be explained as follows: (a) CaMKII might activate another kinase that in turn phosphorylates HDAC5 and, therefore, regulates HDAC5 indirectly, possibly in a cell type-specific manner; (b) we have evidence that HDAC4 can confer CaMKII responsiveness to HDAC5 via dimerization between the 2 HDACs. The latter concept is currently under further investigation in our laboratory.

Therapentic implications. The results of the present study reveal a unique role of HDAC4 among class IIa HDACs. CaMKII is the only kinase identified thus far to signal specifically one class IIa HDAC. In light of the variety of biological functions of class IIa HDACs, CaMKII is a likely candidate that mediates specificity in the regulation of HDAC4. In particular, as shown in this study, endogenous CaMKII causes localization of HDAC4 to the cytosol of cardiomyocytes whereas a CaMKII signal-resistant HDAC4 mutant blocks agonist-induced cardiomyocyte hypertrophy. The molecular basis of the CaMKII/HDAC4 partnership was pinpointed to a CaMKII- 
docking site on HDAC4. These findings will facilitate screening efforts to identify drug-like molecules that interrupt specifically this interaction. Because CaMKII is also involved in important biological functions such as muscle contractility, memory, and fertilization, an inhibitor specific for the HDAC4/CaMKII interaction but not for the interaction of CaMKII with other partners would potentially bypass biologically essential functions of CaMKII. Efforts to identify such target-specific inhibitors are underway.

\section{Methods}

Chemical reagents and plasmids. PE was purchased from Sigma-Aldrich. KN93, KN62, AIPII-2, staurosporine, Bis, and Gö6976 were obtained from Calbiochem; leptomycin B and PMA were purchased from LC Laboratories. Animals were obtained from Harlan. All animal procedures for these experiments were approved by the University of Texas Southwestern Medical Center Institutional Animal Care and Use Committee.

Epitope-tagged derivates of the CaMKIID splicing variants A, B, and C, CaMKII $\gamma \mathrm{A}, \mathrm{CaMKII} \alpha \mathrm{A}, \mathrm{CaMKII} \beta$ 'e and PKD1 containing amino-terminal Myc tags were generated using the pcDNA3 expression vector (Invitrogen). HDAC7 was fused to an amino-terminal FLAG tag (pcDNA3), and HDAC4 to a carboxy-terminal GFP tag (EGFPN1; Clontech). Epitope-tagged derivatives of constitutively active CaMKI (created by a deletion of the autoinhibitory domain at the $\mathrm{C}$ terminus), HDAC4, HDAC5, and MITR containing HA, FLAG, Myc, or GFP tags were described previously $(6,7,12,15,49)$. Point mutations were introduced with the QuikChange kit (Stratagene). Deletion mutants of HDAC4 were generated by PCR with PFU Turbo polymerase (Stratagene).

Cell culture and transfection assays. COS cells were maintained in DMEM with FBS (10\%), L-glutamine ( $2 \mathrm{mM})$, and penicillin-streptomycin. Transfection of COS cells was performed with FuGENE 6 (Roche Diagnostics) according to manufacturer's instructions.

Indirect immunofluorescence. COS cells were grown on glass coverslips, fixed in paraformaldehyde (4\%), permeabilized in $0.1 \%$ Triton X-100 and blocked in PBS containing goat serum (5\%). Primary antibodies against FLAG (monoclonal or rabbit; Sigma-Aldrich), Myc (polyclonal, A-14; Santa Cruz Biotechnology Inc.), or HA (polyclonal, Y-11; Santa Cruz Biotechnology Inc.) were used at a dilution of 1:200. Secondary antibodies conjugated to either fluorescein or Texas red (Vector Laboratories) were also used at a dilution of 1:200. NRVMs were stained with antibodies directed against sarcomeric $\alpha$-actinin (Sigma-Aldrich) and ANP (Bachem). All images were captured at a magnification of $\times 40$.

Coimmunoprecipitation and immunoblotting. COS cells were harvested 1 day after transfection in Tris (50 mM, pH 7.4), NaCl (150-900 mM), EDTA $(1 \mathrm{mM})$, and Triton X-100 (1\%) or in RIPA buffer supplemented with protease inhibitors (Complete; Roche Diagnostics) and PMSF (1 mM). Cells were further disrupted by passage through a 25 -gauge needle and cell debris removed by centrifugation. FLAG-tagged proteins were immunoprecipitated with M2-agarose conjugate (Sigma-Aldrich) and thoroughly washed with lysis buffer. Bound proteins were resolved by SDS-PAGE, transferred to PVDF membranes, and immunoblotted as indicated with either anti-Myc antibody (polyclonal, A-14; Santa Cruz Biotechnology Inc.), a monoclonal anti-FLAG antibody (M2; Sigma-Aldrich), or a polyclonal anti-14-3-3 antibody (Abcam). Proteins were visualized with a chemiluminescence system (Santa Cruz Biotechnology Inc.).

Mammalian 2-hybrid analysis. A mammalian expression vector encoding the GAL4 DNA-binding domain fused to the amino-terminus of human HDAC4 (amino acids 2-740) was generated in the pM expression vector (Clontech). COS cells were transiently transfected with vectors for GAL4HDAC4, VP16-14-3-3, and a luciferase reporter gene under the control of 5 copies of a GAL4 DNA-binding site $(5 \times$ UAS-luciferase $)$ in the absence or presence of a construct encoding constitutively active CaMKIISB. Transfection efficiency was controlled by cotransfection of CMV-lacZ. Twenty-four hours after transfection, cells were harvested and luciferase and $\beta$-galactosidase levels were determined as described above.

GST pull-down assay. DNA fragments encoding amino acids 419-670 of WT HDAC4 and the R601F mutant of HDAC4 were subcloned into the pGEX-4T3 vector. A total of $500 \mathrm{ng}$ of active His-tagged CaMKII $8 \mathrm{~B}$ (His-CaMKIIסB; Upstate USA Inc.) was incubated with $1 \mu \mathrm{g}$ of agarose bead-bound GST, GST-HDAC4-WT, or GST-HDAC4-R601F fusion protein in $300 \mu \mathrm{l}$ of GST-binding buffer $(20 \mathrm{mM}$ Tris $\mathrm{pH} 7.4,150 \mathrm{mM} \mathrm{NaCl}$, $0.5 \%$ NP-40, and protease inhibitor cocktail; Roche Diagnostics) for 1 hour at $4^{\circ} \mathrm{C}$. The beads were washed 3 times in $800 \mu$ l of GST-binding buffer; the bound proteins were resolved by SDS-PAGE and detected by Western blot using an anti-His antibody (Upstate USA Inc.). Loading of GST fusion proteins was confirmed by Coomassie staining of the PVDF membrane after Western blot analysis.

In vitro kinase assay. A total of $500 \mathrm{ng}$ of agarose bead-bound GSTHDAC4-WT or GST-HDAC4-R601F fusion protein was incubated alone or with His-CaMKIII in $30 \mu \mathrm{l}$ of in vitro kinase incubation buffer $(50 \mathrm{mM}$ HEPES/KOH, pH 7.4, $10 \mathrm{mM} \mathrm{MgCl}, 1 \mathrm{mM} \mathrm{CaCl}_{2}, 0.6 \mu \mathrm{M}$ calmodulin, $6 \mu \mathrm{M} \mathrm{ATP}, 500 \mathrm{mM} \mathrm{NaCl}_{2}$, and $0.2 \mu \mathrm{Ci}\left[\gamma^{-32} \mathrm{P}\right]$-ATP) for 10 minutes at $30^{\circ} \mathrm{C}$. In control experiments, $\mathrm{CaCl}_{2}$ and calmodulin were admitted and $1 \mathrm{mM}$ EGTA was added. The reaction volume was resolved by SDS-PAGE, and ${ }^{32} \mathrm{P}$ autoradiography was performed.

Cardiomyocyte culture and adenoviral infection. NRVMs were isolated from 1- to 2-day-old Sprague-Dawley rats as previously described (50). For adenovirus production, cDNAs encoding FLAG-tagged HDAC4-WT or a HDAC4-S246,467,632A were subcloned into the $\mathrm{p}-\mathrm{ACCMV}$ vector and cotransfected with p-JM17 into 293 cells. Primary lysates were used to reinfect 293 cells, and viral plaques were obtained using the agar overlay method. The production of adenoviral GFP-HDAC5 was described previously (16). After isolation, NRVMs were maintained in DMEM:199 medium (4:1) with FBS (10\%), L-glutamine ( $2 \mathrm{mM})$, and penicillin-streptomycin. NRVMs were infected 24 hours after plating, grown 12 hours later in serum-free media for another 4 hours, and then stimulated with $\mathrm{PE}$ for the indicated time period. In experiments with kinase inhibitors, NRVMs were pretreated with the inhibitor 30 minutes prior to stimulation. For assessing cardiomyocyte hypertrophy, NRVMs were grown in serum-free medium for 24 hours and stimulated with PE for another 24-48 hours. Subcellular localization of FLAG-HDAC4, ANP, and cardiomyocyte hypertrophy, as assessed by sarcomeric organization, were determined by indirect immunofluorescence. Images were obtained with a Zeiss LSM 510-Meta confocal microscope or a Leica Microsystems DMRXE compound microscope.

$\left[{ }^{3} \mathrm{H}\right]$-leucine incorporation. Protein synthesis was examined by analysis of $\left[{ }^{3} \mathrm{H}\right]$-leucine incorporation. Two hours after the beginning of PE stimulation of adHDAC4-WT or -S246,467,632A infected NRVMs, $3 \mu \mathrm{l} \mathrm{Ci}$ of $\left[{ }^{3} \mathrm{H}\right]$-leucine was added, and the cells were cultured for an additional 24 hours. After 2 washes with ice-cold PBS, NRVMs were precipitated with $10 \%$ trichloroacetic acid for 30 minutes at $4^{\circ} \mathrm{C}$. The precipitates were then solubilized in $0.2 \mathrm{~N} \mathrm{NaOH}$ for more than 4 hours. Radioactivity was measured in a liquid scintillation counter.

Statistics. Results are expressed as mean \pm SEM. Differences between groups were tested for statistical significance using the unpaired 2-tailed Student's $t$ test. $P$ values less than 0.05 were considered significant.

\section{Acknowledgments}

We thank Timothy McKinsey, Christopher Antos, and Rhonda Bassel-Duby for advice, Igor Rybkin for help with confocal microscopy, and Robert Gerard for adenovirus production. We are grate- 
ful to Mi-Sung Kim, Rick Vega, and Steve R. Grant for plasmids and Alisha Tizenor for assistance with graphics. This work is supported by grants from the NIH, the Donald W. Reynolds Center for Clinical Cardiovascular Research, the Muscular Dystrophy Association, the Robert A. Welch Foundation, the Texas Advanced Technology Program (to E.N. Olson), and the Deutsche Forschungsgemeinschaft (BA 2258/1-1 to J. Backs).
Received for publication November 17, 2005, and accepted in revised form April 18, 2006.

Address correspondence to: Eric N. Olson, University of Texas Southwestern Medical Center, 5323 Harry Hines Boulevard, Dallas, Texas 75390-9148, USA. Phone: (214) 648-1187; Fax: (214) 648-1196; E-mail: eric.olson@utsouthwestern.edu.
1. Roth, S.Y., Denu, J.M., and Allis, C.D. 2001. Histone acetyltransferases. Annu. Rev. Biochem. 70:81-120.

2. Grozinger, C.M., and Schreiber, S.L. 2002. Deacetylase enzymes: biological functions and the use of small-molecule inhibitors. Chem. Biol. 9:3-16.

3. Backs, J., and Olson, E.N. 2006. Control of cardiac growth by histone acetylation/deacetylation. Circ. Res. 98:15-24.

4. Verdin, E., Dequiedt, F., and Kasler, H.G. 2003. Class II histone deacetylases: versatile regulators. Trends Genet. 19:286-293.

5. Grozinger, C.M., and Schreiber, S.L. 2000. Regulation of histone deacetylase 4 and 5 and transcriptional activity by 14-3-3-dependent cellular localization. Proc. Natl. Acad. Sci. U. S. A. 97:7835-7840.

6. McKinsey, T.A., Zhang, C.L., Lu, J., and Olson, E.N. 2000. Signal-dependent nuclear export of a histone deacetylase regulates muscle differentiation. Nature. 408:106-111.

7. McKinsey, T.A., Zhang, C.L., and Olson, E.N. 2001. Identification of a signal-responsive nuclear export sequence in class II histone deacetylases. Mol. Cell. Biol. 21:6312-6321.

8. Edmondson, D.G., Lyons, G.E., Martin, J.F., and Olson, E.N. 1994. Mef2 gene expression marks the cardiac and skeletal muscle lineages during mouse embryogenesis. Development. 120:1251-1263.

9. Passier, R., et al. 2000. CaM kinase signaling induces cardiac hypertrophy and activates the MEF2 transcription factor in vivo. J. Clin. Invest. 105:1395-1406.

10. Naya, F.J., Wu, C., Richardson, J.A., Overbeek, P., and Olson, E.N. 1999. Transcriptional activity of MEF2 during mouse embryogenesis monitored with a MEF2-dependent transgene. Development. 126:2045-2052.

11. Miska, E.A., et al. 1999. HDAC4 deacetylase associates with and represses the MEF2 transcription factor. $E M B O$ J. 18:5099-5107.

12. Zhang, C.L., et al. 2002. Class II histone deacetylases act as signal-responsive repressors of cardiac hypertrophy. Cell. 110:479-488.

13. Chang, S., et al. 2004. Histone deacetylases 5 and 9 govern responsiveness of the heart to a subset of stress signals and play redundant roles in heart development. Mol. Cell. Biol. 24:8467-8476.

14. Vega, R.B., et al. 2004. Histone deacetylase 4 controls chondrocyte hypertrophy during skeletogenesis. Cell. 119:555-566.

15. McKinsey, T.A., Zhang, C.L., and Olson, E.N. 2000. Activation of the myocyte enhancer factor- 2 transcription factor by calcium/calmodulin-dependent protein kinase-stimulated binding of 14-3-3 to histone deacetylase 5. Proc. Natl. Acad. Sci. U. S. A. 97:14400-14405.

16. Vega, R.B., et al. 2004. Protein kinases C and D mediate agonist-dependent cardiac hypertrophy through nuclear export of histone deacetylase 5 . Mol. Cell. Biol. 24:8374-8385.

17. Dequiedt, F., et al. 2005. Phosphorylation of histone deacetylase 7 by protein kinase D mediates $T$ cell receptor-induced Nur77 expression and apoptosis. J. Exp. Med. 201:793-804.

18. Harrison, B.C., et al. 2006. Regulation of cardiac stress signaling by protein kinase d1. Mol. Cell. Biol. 26:3875-3888.

19. Davis, F.J., Gupta, M., Camoretti-Mercado, B., Schwartz, R.J., and Gupta, M.P. 2003. Calcium/ calmodulin-dependent protein kinase activates serum response factor transcription activity by its dissociation from histone deacetylase, HDAC4. Implications in cardiac muscle gene regulation during hypertrophy. J. Biol. Chem. 278:20047-20058.

20. Zhao, X., et al. 2001. The modular nature of histone deacetylase HDAC4 confers phosphorylationdependent intracellular trafficking. J. Biol. Chem. 276:35042-35048.

21. Liu, Y., Randall, W.R., and Schneider, M.F. 2005. Activity-dependent and -independent nuclear fluxes of HDAC4 mediated by different kinases in adult skeletal muscle. J. Cell Biol. 168:887-897.

22. Bolger, T.A., and Yao, T.P. 2005. Intracellular trafficking of histone deacetylase 4 regulates neuronal cell death. J. Neurosci. 25:9544-9553.

23. Srinivasan, M., Edman, C.F., and Schulman, H. 1994. Alternative splicing introduces a nuclear localization signal that targets multifunctional CaM kinase to the nucleus. J. Cell Biol. 126:839-852.

24. Heist, E.K., Srinivasan, M., and Schulman, H. 1998. Phosphorylation at the nuclear localization signal of $\mathrm{Ca} 2+/$ calmodulin-dependent protein kinase II blocks its nuclear targeting. J. Biol. Chem. 273:19763-19771.

25. Chang, S., Bezprozvannaya, S., Li, S., and Olson, E.N. 2005. An expression screen reveals modulators of class II histone deacetylase phosphorylation. Proc. Natl. Acad. Sci. U. S. A. 102:8120-8125.

26. Heist, E.K., and Schulman, H. 1998. The role of $\mathrm{Ca} 2+/$ calmodulin-dependent protein kinases within the nucleus. Cell Calcium. 23:103-114.

27. Maier, L.S., and Bers, D.M. 2002. Calcium, calmodulin, and calcium-calmodulin kinase II: heartbeat to heartbeat and beyond. J. Mol. Cell. Cardiol. 34:919-939.

28. Wu, X., et al. 2006. Local InsP3-dependent perinuclear $\mathrm{Ca}_{2}{ }^{+}$signaling in cardiac myocyte excitationtranscription coupling. J. Clin. Invest. 116:675-682. doi:10.1172/JCI27374.

29. Xu, X., et al. 2005. ASF/SF2-regulated CaMKIIdelta alternative splicing temporally reprograms excitation-contraction coupling in cardiac muscle. Cell. 120:59-72.

30. Colomer, J.M., Mao, L., Rockman, H.A., and Means, A.R. 2003. Pressure overload selectively up-regulates $\mathrm{Ca} 2+/$ calmodulin-dependent protein kinase II in vivo. Mol. Endocrinol. 17:183-192.

31. Hudmon, A., and Schulman, H. 2002. Structurefunction of the multifunctional $\mathrm{Ca} 2+/$ calmodulin-dependent protein kinase II. Biochem. J. 364:593-611.

32. Tombes, R.M., Faison, M.O., and Turbeville, J.M. 2003. Organization and evolution of multifunctional $\mathrm{Ca}(2+) / \mathrm{CaM}$-dependent protein kinase genes. Gene. 322:17-31.

33. Michel, J.J., and Scott, J.D. 2002. AKAP mediated signal transduction. Annu. Rev. Pharmacol. Toxicol. 42:235-257.

34. Bayer, K.U., Harbers, K., and Schulman, H. 1998. alphaKAP is an anchoring protein for a novel CaM kinase II isoform in skeletal muscle. EMBO J. 17:5598-5605

35. Leonard, A.S., et al. 2002. Regulation of calcium/ calmodulin-dependent protein kinase II docking to N-methyl-D-aspartate receptors by calcium/calmodulin and alpha-actinin. J. Biol. Chem. 277:48441-48448.
36. Zhang, T., and Brown, J.H. 2004. Role of Ca2+/ calmodulin-dependent protein kinase II in cardiac hypertrophy and heart failure. Cardiovasc. Res. 63:476-486.

37. Hoch, B., Meyer, R., Hetzer, R., Krause, E.G., and Karczewski, P. 1999. Identification and expression of delta-isoforms of the multifunctional $\mathrm{Ca} 2+/$ calmodulin-dependent protein kinase in failing and nonfailing human myocardium. Circ. Res. 84:713-721.

38. Zhang, R., et al. 2005. Calmodulin kinase II inhibition protects against structural heart disease. Nat. Med. 11:409-417.

39. Ramirez, M.T., Zhao, X.L., Schulman, H., and Brown, J.H. 1997. The nuclear deltaB isoform of $\mathrm{Ca} 2+/$ calmodulin-dependent protein kinase II regulates atrial natriuretic factor gene expression in ventricular myocytes. J. Biol. Chem. 272:31203-31208.

40. Colomer, J.M., and Means, A.R. 2000. Chronic elevation of calmodulin in the ventricles of transgenic mice increases the autonomous activity of calmodulin-dependent protein kinase II, which regulates atrial natriuretic factor gene expression. Mol. Endocrinol. 14:1125-1136.

41. Zhu, W., et al. 2000. Ca2+/calmodulin-dependent kinase II and calcineurin play critical roles in endothelin-1-induced cardiomyocyte hypertrophy. J. Biol. Chem. 275:15239-15245.

42. Gruver, C.L., DeMayo, F., Goldstein, M.A., and Means, A.R. 1993. Targeted developmental overexpression of calmodulin induces proliferative and hypertrophic growth of cardiomyocytes in transgenic mice. Endocrinology. 133:376-388.

43. Zhang, T., et al. 2002. The cardiac-specific nuclear delta(B) isoform of $\mathrm{Ca} 2+/$ calmodulin-dependent protein kinase II induces hypertrophy and dilated cardiomyopathy associated with increased protein phosphatase 2A activity. J. Biol. Chem. 277:1261-1267.

44. Zhang, T., et al. 2003. The deltaC isoform of CaMKII is activated in cardiac hypertrophy and induces dilated cardiomyopathy and heart failure. Circ. Res. 92:912-919.

45. Lint, J.V., Rykx, A., Vantus, T., and Vandenheede, J.R. 2002. Getting to know protein kinase D. Int. J. Biochem. Cell Biol. 34:577-581.

46. Dequiedt, F., et al. 2003. HDAC7, a thymus-specific class II histone deacetylase, regulates Nur77 transcription and TCR-mediated apoptosis. Immunity. 18:687-698.

47. Chawla, S., Vanhoutte, P., Arnold, F.J., Huang, C.L., and Bading, H. 2003. Neuronal activity-dependent nucleocytoplasmic shuttling of HDAC4 and HDAC5. J. Neurochem. 85:151-159.

48. Linseman, D.A., et al. 2003. Inactivation of the myocyte enhancer factor- 2 repressor histone deacetylase- 5 by endogenous $\mathrm{Ca}(2+) / /$ calmodulin-dependent kinase II promotes depolarizationmediated cerebellar granule neuron survival. J. Biol. Chem. 278:41472-41481.

49. Zhang, C.L., McKinsey, T.A., Lu, J.R., and Olson, E.N. 2001. Association of COOH-terminal-binding protein (CtBP) and MEF2-interacting transcription repressor (MITR) contributes to transcriptional repression of the MEF2 transcription factor. J. Biol. Chem. 276:35-39.

50. Molkentin, J.D., et al. 1998. A calcineurin-dependent transcriptional pathway for cardiac hypertrophy. Cell. 93:215-228. 